\title{
Deciphering the contribution of lipid droplets in leprosy: multifunctional organelles with roles in Mycobacterium leprae pathogenesis
}

\author{
Katherine Antunes de Mattos ${ }^{1}$, Euzenir Nunes Sarno², \\ Maria Cristina Vidal Pessolani', Patricia T Bozza ${ }^{3 /+}$ \\ ${ }^{1}$ Laboratório de Microbiologia Celular ${ }^{2}$ Laboratório de Hanseníase \\ ${ }^{3}$ Laboratório de Imunofarmacologia, Instituto Oswaldo Cruz-Fiocruz, Rio de Janeiro, RJ, Brasil
}

\begin{abstract}
Leprosy is an infectious disease caused by Mycobacterium leprae that affects the skin and nerves, presenting a singular clinical picture. Across the leprosy spectrum, lepromatous leprosy (LL) exhibits a classical hallmark: the presence of a collection of M. leprae-infected foamy macrophages/Schwann cells characterised by their high lipid content. The significance of this foamy aspect in mycobacterial infections has garnered renewed attention in leprosy due to the recent observation that the foamy aspect represents cells enriched in lipid droplets (LD) (also known as lipid bodies). Here, we discuss the contemporary view of $L D$ as highly regulated organelles with key functions in $\mathrm{M}$. leprae persistence in the LL end of the spectrum. The modern methods of studying this ancient disease have contributed to recent findings that describe $\mathrm{M}$. leprae-triggered LD biogenesis and recruitment as effective mycobacterial intracellular strategies for acquiring lipids, sheltering and/or dampening the immune response and favouring bacterial survival, likely representing a fundamental aspect of $\mathrm{M}$. leprae pathogenesis. The multifaceted functions attributed to the $L D$ in leprosy may contribute to the development of new strategies for adjunctive antileprosy therapies.
\end{abstract}

Key words: leprosy - lipid droplet - eicosanoids - bacterial survival - pathogenicity - nutritional source - inflammation

Leprosy, or Hansen's disease, is an ancient chronic infectious disease caused by Mycobacterium leprae, a slow-growing obligate intracellular bacterial pathogen. Despite the "elimination of leprosy" slogan that has dominated the priorities of leprosy work and the success of multidrug therapy (MDT), which has succeeded in reducing the leprosy burden over the years, this disease remains a public health problem in several countries (WHO 2010). The fact that the elimination of leprosy has not yet been achieved suggests that the infection has not been adequately controlled by antibiotics alone. Thus, it is critical to invest in broad-based research studies that examine the interaction of $M$. leprae with host cells to identify new approaches to improve our understanding of the pathophysiology of leprosy and to identify new therapeutic targets.

Leprosy manifests as a spectrum of clinical forms dictated by the type and magnitude of the immunological response mounted by the host against the infection. The tuberculoid (TT) and lepromatous (LL) polar clinical forms are at the two extremes of the spectrum, whereas the intermediate clinical forms present with hybrid characteristics (Ridley 1974). TT patients develop a specific, strong T-helper (Th)1-type response, which restricts $M$. leprae multiplication and dissemination and limits the

Financial support: IOC/FIOCRUZ, CNPq, FAPERJ, CAPES

PTB is a Guggenheim fellow.

+ Corresponding author: pbozza@ioc.fiocruz.br

Received 25 May 2012

Accepted 30 August 2012 disease to a few lesions. In contrast, LL is a progressive and disseminated disease that is characterised by extensive bacterial multiplication within the host cells and a Th2-like profile in the lesions (Parkash 2009). The German physician and pathologist Rudolf Virchow (1821-1902) described lipid-accumulating macrophages in biopsies from LL lesions and these macrophages, known as Lepra cells (or foamy cells), are distinctive for this severe form of leprosy (Virchow 1863). Later, the presence of collections of highly infected Schwann cells with the same phenotype were also described in nerve biopsies of LL patients, but not TT patients (Job 1970) (Fig. 1A). A close examination of these cells suggested that $M$. leprae resides and replicates within enlarged, lipid-filled phagosomes (Chatterjee et al. 1959), indicating an important alteration in lipid metabolism during infection. However, the mechanism(s) that disturbs the lipid homeostasis of $M$. leprae-infected cells, in addition to the origin, nature and functions of that disturbance, has remained unclear. The lipids were initially thought to be derived from M. leprae (Sakurai \& Skinsnes 1970); however, independent studies have provided evidence that the foamy aspect is, at least in part, derived from host lipids, such as oxidised phospholipids and cholesterol esters (Kurup \& Mahadevan 1982, Cruz et al. 2008, Mattos et al. 2010). These data are reinforced by recent microarray data analyses that genes associated with lipid metabolism are upregulated after $M$. leprae infection, particularly among LL patients (Cruz et al. 2008), as also described during the shift from latent to active tuberculosis (TB) (Kim et al. 2010). Moreover, D'Avila et al. (2006) have demonstrated that the foamy aspect of mycobacteria-infected cells is mostly due to pathogentriggered host lipid droplets (LD). 
Contemporary evidence supports a key role for the regulation of lipid metabolism and LD in the host response to intracellular pathogens; also termed lipid bodies or adiposomes, LDs are dynamic lipid-enriched organelles found in different cell types. Pathogen-induced increases in LD formation have been described in bac- terial, viral, fungal and parasitic infections and a role for this organelle in intracellular survival and pathogen replication has been proposed for all of these infections (reviewed in D'Avila et al. 2008a, Bozza et al. 2009a, van der Meer-Janssen et al. 2010). Therefore, the aim of this review article is to discuss the roles of LD in $M$.

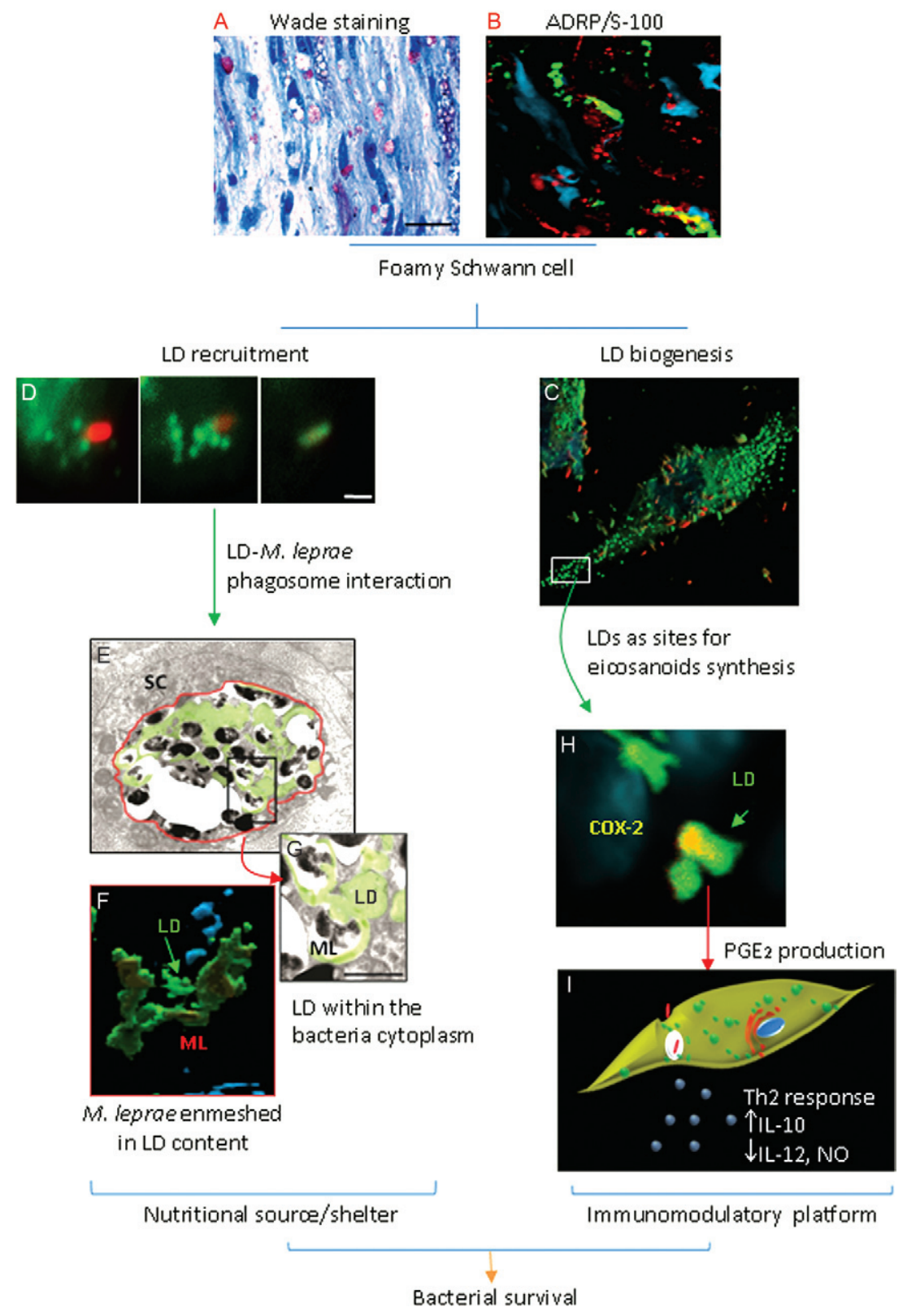

Fig. 1: overview of lipid droplet (LD) function in leprosy disease. Foamy phenotype from a lepromatous leprosy nerve visualized by Wade staining (A) and immunohistochemistry confirming the LD accumulation in Schwann cells (SC) using anti-adipose differentiation-related protein (ADRP) and anti-S-100 antibodies (B). In vitro model of Mycobacterium leprae (ML)-trigger biogenesis (C) and recruitment (D). Lipid recruitment culminates in LD-M. leprae phagosome interaction (E) with consequent enmeshment of bacilli in LD (F) and possible incorporation of this lipid content into the bacterial cytoplasm $(\mathrm{G})$. This sequence of events suggests the role of LD as a nutritional source and/or shelter in the host cell. In addition, LD organelles compartmentalize the cyclooxygenase (COX)-2 enzyme (H), reinforcing its role as an immunoinflammatory platform to prostaglandin $\mathrm{E} 2\left(\mathrm{PGE}_{2}\right.$ ) production and upregulation of T-helper (Th)2/Th1 cytokines (I). Together, these data suggest LDs are multifunctional organelles in leprosy disease that contribute to bacterial survival. IL: interleukin; NO: nitric oxide. Fig. 1A-C, with permission from Mattos et al. (2011b) (C2011 The American Association of Immunologists, Inc), C-F, with permission from Mattos et al. (2011a) (C2011 The Wiley Online Library). 
leprae infection, focusing on the mechanisms of lipid droplet formation and lipid droplet composition and function within the context of leprosy. Indeed, recent findings have demonstrated that LDs are highly induced in a regulated manner during $M$. leprae infection. The LD formed in leprosy are emerging as organelles with major functions in disease pathogenesis, including roles in pathogen evasion and as a rich source of nutrients that allow the persistence of M. leprae.

Lipid droplet biogenesis - Lipid droplet biogenesis is a biological phenomenon that has gained attention over the last few years, mainly due to its intimate relationships with inflammatory and infection processes. Although different hypotheses regarding lipid droplet biogenesis have been described and the subject is still highly controversial, there is a consensus that both pathogen and host factors govern the molecular mechanisms of this process. Renewed attention is being given to the formation of LD within host cells infected by intracellular pathogens, a phenomenon that has been observed for all of the four main groups of pathogens (i.e., viruses, bacteria, fungi and parasites) (Bozza et al. 2009a, b, van der Meer Janssen et al. 2010, Saka \& Valdivia 2012). In most cases, it appears that the pathogen takes advantage of the LD formed to support its own life cycle. This intriguing concept points to new, previously unrecognised aspects of lipid droplet biology. In this section, we focus on the current knowledge of the mechanisms that regulate lipid droplet formation during the host response to M. leprae.

The pathogen-triggered dysregulation of host cell lipid metabolism is an emerging feature in the pathogenesis of mycobacterial infections. Accumulating evidence suggests that mycobacterial pathogenesis is directly related to the modulation of host lipid metabolism. During the infectious process, LDs are directly triggered to form by pathogenic mycobacterial species, including $\mathrm{Myco-}$ bacterium bovis (D'Avila et al. 2006, 2008b, Almeida et al. 2009), Mycobacterium tuberculosis (Peyron et al. 2008, Cáceres et al. 2009) and M. leprae (Tanigawa et al. 2008, Mattos et al. 2010, 2011a, b); in contrast, such non-pathogenic species as Mycobacterium smegmatis are not able to induce these organelles (D'Avila et al. 2006, Peyron et al. 2008, Almeida et al. 2009, Mattos et al. 2010). However, as summarised in Table, differential mechanisms govern this phenomenon. This differential profile of lipid droplet induction among mycobacteria may be related to the obligate intracellular lifestyle and the levels of pathogenicity of these species, reinforcing the idea that lipid accumulation is intimately related to bacterial survival and persistence in the host cell.

Regarding leprosy, in particular, our own studies, which have focused on the lipid accumulation in macrophages, as associated with the cutaneous lesions of LL, have revealed that these foamy-appearing, lipid-laden cells originate, at least in part, from the lipid droplet formation triggered by an M. leprae infection. The mechanisms that govern the lipid droplet formation process in M. leprae-infected macrophages have been investigated using in vitro models (human and murine macrophages). These mechanisms exhibit similarities to M. bovis BCG infection during which lipid droplet biogenesis is induced (i) in a time and dose-dependent manner, (ii) by both live and dead mycobacteria, (iii) by mycobacterial cell wall components and (iv) in bacterium-associated

TABLE

Differential regulatory mechanism of mycobacteria-trigger host lipid droplet (LD) biogenesis and their functions during infection

\begin{tabular}{|c|c|c|c|c|c|c|c|c|}
\hline \multirow[t]{2}{*}{ Mycobacterium sp. } & \multicolumn{2}{|c|}{$\begin{array}{c}\text { Mycobacterium } \\
\text { smegmatis }\end{array}$} & \multicolumn{2}{|c|}{$\begin{array}{c}\text { Mycobacterium } \\
\text { bovis BCG }\end{array}$} & \multicolumn{2}{|c|}{$\begin{array}{c}\text { Mycobacterium } \\
\text { tuberculosis }\end{array}$} & \multicolumn{2}{|c|}{$\begin{array}{c}\text { Mycobacterium } \\
\text { leprae }\end{array}$} \\
\hline & \multicolumn{2}{|c|}{ Avirulent } & \multicolumn{2}{|c|}{ Virulence-attenuated } & \multicolumn{2}{|c|}{ Virulent } & \multicolumn{2}{|c|}{ Virulent } \\
\hline \multicolumn{9}{|c|}{ LD biogenesis/recruitment } \\
\hline Cell type & $\mathrm{M} \varphi$ & $\mathrm{SC}$ & $\mathrm{M \varphi}$ & $\mathrm{SC}$ & $\mathrm{M} \varphi$ & $\mathrm{SC}$ & $\mathrm{M} \varphi$ & $\mathrm{SC}$ \\
\hline Host LD induction & $\mathrm{No}^{a, b}$ & $\mathrm{No}^{b}$ & Yes $^{a}$ & $\mathrm{No}^{c}$ & Yes $^{\mathrm{d}}$ & ND & Yes $^{b}$ & Yes $^{c}$ \\
\hline Bacterial viability dependency & - & - & $\mathrm{No}^{a}$ & - & $\mathrm{No}^{d}$ & - & $\mathrm{No}^{b}$ & Yes $^{c}$ \\
\hline TLR signalling & - & - & TLR2 $^{a}$ & - & ND & - & TLR $2 / 6^{b}$ & TLR6 $^{c}$ \\
\hline Paracrine signalling & - & - & Yes $^{a}$ & - & ND & - & Yes $^{b}$ & $\mathrm{No}^{c}$ \\
\hline $\begin{array}{l}\text { Host LD-mycobacterial } \\
\text { phagosome interaction }\end{array}$ & - & - & Yes $^{a}$ & - & Yes $^{d}$ & - & Yes $^{f}$ & Yes $^{g}$ \\
\hline \multicolumn{9}{|c|}{ LD functions } \\
\hline Immunomodulators & - & - & $\mathrm{PGE}_{2} / \mathrm{LTB}_{4}{ }^{a}$ & - & $\mathrm{PGE}_{2} / \mathrm{LTA}_{4}^{e}$ & - & $\mathrm{PGE}_{2}^{b}$ & $\mathrm{PGE}_{2}^{c}$ \\
\hline Shelter/nutrition & - & - & $\operatorname{Yes}^{a}$ & - & $\operatorname{Yes}^{d, e}$ & - & Yes $^{g}$ & Yes $^{c, g}$ \\
\hline
\end{tabular}

$a$ : D'Avila et al. (2006); b: Mattos et al. (2010); $c$ : Mattos et al. (2011b); $d$ : Peyron et al. (2008); $e$ : Daniel et al. (2011); $f$ : Tanigawa et al. (2008); g: Mattos et al. (2011a); LT: leukotrienes; M $\varphi$ : macrophage; ND: not determined; SC: Schwann cells; PGE: prostaglandin E; TLR: Toll-like receptor. 
cells and in cells with no internalised bacteria, indicating that soluble factors secreted by neighbouring infected cells are able to induce the bystander formation of these organelles (Fig. 2). However, differential lipid droplet-formation signalling induced by these mycobacterial species has also been noted. Whereas lipid droplet formation induced by $M$. bovis BCG involves a Toll-like receptor (TLR)2-dependent signalling process, this phenomenon is induced in a TLR2/TLR6-dependent manner during M. leprae infection (D'Avila et al. 2006, Almeida et al. 2009, Mattos et al. 2010).

Interestingly, we have shown that the mechanisms of lipid droplet induction by M. leprae in Schwann cells share similarities with those observed in macrophages, but also exhibit some differences (Table) (Mattos et al. 2010, 2011b). Lipid droplet biogenesis is induced in a time and dose-dependent manner and requires bacterial phagocytosis. Moreover, using selective drugs, we have

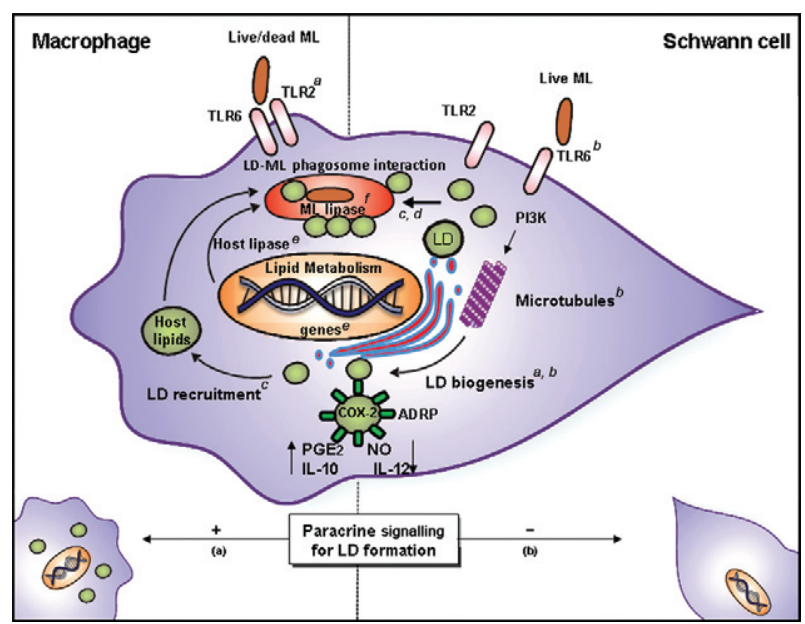

Fig. 2: $a$ : Mattos et al. (2010); $b$ : Mattos et al. (2011b); $c$ : Mattos et al. (2011a); $d$ : Tanigawa et al. (2008); $e$ : Cruz et al. (2008); $f$ : Cole et al. (2001). Molecular mechanisms regulating the Mycobacterium leprae (ML) trigger of lipid droplet (LD) biogenesis/recruitment in host cells. In leprosy, the mechanisms of LD formation by ML in Schwann cells share similarities, but also exhibit some differences compared with those observed in macrophages. Macrophages induced LD in a Toll-like receptor (TLR)2/TLR6 mechanism, independent of bacterial viability and dependent of phagocytosis and paracrine signalling $(a)$. Schwann cells induced LD in a TLR6-dependent and TLR2-independent mechanism, dependent of bacterial viability and phagocytosis and independent of paracrine signalling $(b)$. LDs are promptly recruited to bacterial-containing phagosomes, where they accumulate $(c, d)$. ML-induced LD biogenesis is an endoplasmic reticulum-Golgi and actin or microtubule-dependent event $(b)$. LD recruitment may constitute an intracellular strategy for ML persistence within host cells by subversion of the host lipid metabolism $(c)$. The proposed mechanism of lipid acquisition from LDs is reinforced by the presence of bacterial and host lipases $(d, e)$. In addition, new LDs constitute active catalytic sites of prostaglandin E2 $\left(\mathrm{PGE}_{2}\right)$ synthesis and contribute to the Thelper (Th)2 cytokine profile $(a, b)$. Together, the LD biogenesis/recruitment favours the generation of an innate immune response (Th2) and an environment (shelter/nutritional source) that may contribute to ML persistence. ADRP: adipose differentiation-related protein; COX: cyclooxigenase; IL: interleukin; NO: nitric oxide; PI3K: phosphoinositide 3-kinase. shown that lipid droplet biogenesis in M. leprae-infected Schwann cells follows a classical route. LDs are known to originate in the endoplasmic reticulum (ER)-Golgi complex and the capacity of LD to form and grow in size is dependent on motor proteins and microtubules (Pacheco et al. 2007, Zehmer et al. 2009). The induction of LD in Schwann cells by M. leprae is dependent on the ER and Golgi apparatus because it is inhibited by the drugs brefeldin and monensin, which are known to disrupt the ER-Golgi complex (Fig. 2). In addition, the disturbance of microtubule and actin organisation by specific drugs reveals that $M$. leprae-induced lipid droplet biogenesis also signals through a pathway that is dependent on cytoskeleton dynamics. Lastly, the capacity of M. leprae to induce LD in Schwann cells can be abrogated with inhibitors of phosphoinositide 3-kinase (PI3K), an enzyme that participates in several signalling pathways, including cytoskeletal organisation (Fig. 2) (Mattos et al. 2011a).

However, the induction of LD in Schwann cells exhibits interesting and unique features when compared to induction in macrophages. First, in contrast to macrophages, live $M$. leprae, but not dead bacteria or isolated mycobacterial components, are able to induce LD in in vitro-cultured Schwann cells, suggesting that the bacteria may play an active role in lipid droplet formation in this cell type (Mattos et al. 2011b). Second, in $M$. leprae-infected cultures, M. leprae-induced lipid droplet formation is observed in cells associated with bacteria, but not in cells without internalised bacteria (Mattos et al. 2011b). In deep contrast to the observations in macrophages, a culture medium conditioned by $M$. lepraestimulated Schwann cells is unable to mimic the ability of the bacteria to induce LD, confirming the absence of any soluble factors secreted by infected Schwann cells that are able to promote lipid droplet biogenesis in uninfected neighbouring cells (Mattos et al. 2011a, b) (see below for further discussion). A third important difference between the M. leprae-induced lipid droplet biogenesis in macrophages and Schwann cells is related to the differential requirement of pattern recognition receptors (Mattos et al. 2011a, b), as discussed below.

The role of innate immune receptors in lipid droplet formation - The first level of mycobacterial interaction and recognition by host cells consists of pattern recognition receptors, such as the TLRs. Different members of the TLR family, including TLR1, TLR2, TLR4 and TLR6, have been implicated in mycobacterial recognition (Means et al. 1999, Takeuchi et al. 2002, Krutzik et al. 2003, Oliveira et al. 2003). The stimulation of TLR signalling by bacteria and bacterial molecules has been shown to promote the accumulation of lipids in macrophages in the form of lipid droplet organelles. Experiments performed using macrophages from TLR-deficient mice have revealed that lipid droplet formation in response to bacteria is dependent on TLR signalling, particularly via TLR2 (Pacheco et al. 2002, D’Avila et al. 2006, Cao et al. 2007, Almeida et al. 2009, Mattos et al. 2010, 2011b). Moreover, it has been shown that killed bacteria and purified molecules derived from bacteria are also capable of promoting lipid accumulation in treated macrophages (Pacheco et al. 2002, Kazemi et al. 2005, D'Avila et al. 
2006, Chen et al. 2008, Almeida et al. 2009, Mattos et al. 2010). These findings suggest that the mechanisms responsible for bacteria-induced lipid droplet formation involve the recognition of bacterial pathogen-associated molecular patterns (PAMPs) by TLRs. These data are reinforced by observations that non-virulent bacterial species only induce foamy macrophages after transformation with a gene encoding an enzyme that catalyses the oxygenation of mycolic acids (Russell et al. 2009).

Specifically in leprosy, the role of TLRs in M. leprae-induced lipid droplet biogenesis is unlike the regulation described for BCG and there is differential regulation depending on the cell type and bacterial viability (Table). BCG and the purified cell component lipoarabinomannan are potent inducers of lipid droplet formation in macrophages from wild-type mice, but fail to induce lipid droplet biogenesis in TLR2-deficient mice, though not TLR4 or TLR6-deficient mice, suggesting a role for TLR2 in this mechanism (D'Avila et al. 2006, Almeida et al. 2009, Mattos et al. 2010). Interestingly, the stimulation of macrophages with M. smegmatis does not induce lipid droplet formation under the same conditions in which BCG infection is highly effective (D'Avila et al. 2006, Almeida et al. 2009, Mattos et al. 2010). In particular, we have demonstrated that the formation of LD in response to M. leprae is dependent on TLR2/TLR6 in infected macrophages (Mattos et al. 2010). In addition, although Schwann cells express TLR2 (Oliveira et al. 2003), signalling through this receptor is not essential for lipid droplet biogenesis. Corroborating these data, BCG is unable to trigger lipid droplet formation signalling in Schwann cells, despite the fact that BCG contains the signature molecules recognised by TLR2, reinforcing the exclusion of TLR2-dependent signalling in this cell type. Moreover, although TLR6 is essential for sensing $M$. leprae in both cell types, a potential additional role for TLR6 as a phagocytic receptor has been observed in Schwann cells. Interestingly, the signalling that excludes the TLR2 pathway and involves a TLR6 heterodimer during inflammation in response to microbial infection has only just been explored for the first time, but it has also recently been described in scenarios of sterile inflammation, such as in atherosclerosis and Alzheimer's disease (Stewart et al. 2010). The question of whether other coreceptors in conjunction with TLR6 participate in the recognition and signalling of live $M$. leprae in Schwann cells deserves further investigation. Additionally, the specific $M$. leprae chemical signatures recognised by TLR6 should be characterised.

Given the importance of TLR-mediated signalling for host resistance to microbial pathogens, much of the research exploring the influence of the genetic background on leprosy has focused on polymorphisms in various TLR genes (Alcais et al. 2005, Cardoso et al. 2011) or other PAMP receptors (de Messias et al. 2009). Genetic factors almost certainly also play a key role in determining whether an infection leads to the TT form of the disease, the LL form of the disease or an intermediate manifestation. In view of the discussed findings, it would not be surprising to discover that the genetic variations that control the tendency to form LD in macrophages/Schwann cells might also be quite important in the foamy phenotype.

Paracrine signalling - The recent observation that the secreted factors from macrophages that stimulate through TLRs also contribute to foamy cell formation suggests that a paracrine or autocrine signal may be involved in this phenomenon, which is of particular interest in the study of foam cell biogenesis (Pacheco et al. 2002, 2007, D'Avila et al. 2006, Montoya et al. 2009). Specifically, it was shown that LD form both in the macrophages that take up $M$. leprae in a TLR2-dependent manner and in neighbouring macrophages that do not take up the bacteria (Mattos et al. 2010). Moreover, the supernatant from challenged macrophages promotes lipid droplet formation in unchallenged macrophages in a TLR2-independent manner, indicating that lipopeptide carryover cannot account for this finding (Mattos et al. 2010). A secondary autocrine mechanism of lipid droplet formation may help to explain a number of paradoxes in current lipid droplet research. In contrast to macrophages, media conditioned by M. leprae-stimulated Schwann cells is unable to replicate the lipid droplet induction observed with bacteria, confirming the absence of soluble factors secreted by infected Schwann cells that are able to promote lipid droplet biogenesis in uninfected neighbouring cells (Table) (Mattos et al. 2011b).

As reviewed extensively elsewhere, mycobacterial infection modulates the production of a number of cytokines by macrophages (Moreno \& Rees 1993). In addition, it has been previously described that cytokines and chemokines may participate in the signalling that leads to lipid droplet formation (Bozza et al. 1998, Halvorsen et al. 2005, Pacheco et al. 2007). To define potential candidates involved in paracrine signalling, we assessed the levels of various cytokines/chemokines in conditioned media from $M$. leprae-infected macrophages. Interestingly, we found a positive correlation between the interleukin (IL)-10/IL-12 ratio and the ability of a conditioned medium to induce lipid droplet formation in uninfected cells (Mattos et al. 2010, 2011b). This observation correlates with the characteristic pattern of IL-10 predominance in LL lesions (Yamamura et al. 1991). Moreover, IL-10 has been recently implicated in foam cell formation in atherosclerosis disease, which reinforces the potential in vivo role of the cytokine balance in the generation and maintenance of abundant LD in LL lesions (Montoya et al. 2009). Additionally, there are significantly higher levels of IL-1 $\beta$ in a conditioned medium that is capable of inducing $\mathrm{LD}$, suggesting a potential role for this cytokine in promoting intracellular lipid storage, as has been recently noted (Persson et al. 2008). However, although an increase in IL-10 has also been observed in conditioned media from M. leprae-infected Schwann cells, this stimulus is insufficient to induce in Schwann cells the paracrine mechanism of lipid droplet biogenesis that is observed in macrophages. These data demonstrate that an M. leprae stimulus leads to distinct host responses that are responsible for the amplification of lipid droplet biogenesis. These responses depend on 
the direct interaction between the pathogen and host cell and also on indirect mechanisms in which neighbouring cells sense an infection through a bystander amplification-induced system triggered by bacterial components and/or host generated cytokines and chemokines.

M. leprae takes advantage of lipid droplet induction for its persistence - The modulation of the host lipid pathway during host-pathogen interactions and virulence will be discussed in this section. Overall, a model can be proposed in which the lipid storage phenomenon observed in $M$. leprae-infected cells plays a key role in leprosy pathogenesis by facilitating bacterial persistence in the host via at least two mechanisms (Fig. 1). First, during $M$. leprae attachment and internalisation, the LDs are promptly recruited to and accumulate in bacteria-containing phagosomes (Mattos et al. 2011a). Lipid droplet recruitment likely constitutes an effective $M$. leprae intracellular strategy for acquiring host cell lipids as a nutritional source and/or shelter to promote bacterial survival. Second, the accumulation of hostderived lipids in infected cells favours the generation of an innate immune response that may contribute to a permissive environment for $M$. leprae proliferation within lepromatous skin and nerves.

Host $L D$ as shelter and/or nutrition for M. leprae We propose that the foamy phenotype (Fig. 1A) observed in LL is a consequence of lipid droplet biogenesis (Fig. 1B, C) and that lipid droplet recruitment (Fig. 1D) may constitute an effective $M$. leprae intracellular strategy for acquiring host lipids and promoting bacterial survival. Blocking lipid droplet formation enables infected cells to kill the invading bacteria, supporting a model in which induced lipid droplet biogenesis is part of the bacterial strategy for persistent infection (Fig. 1C) (Mattos et al. 2011b). Indeed, we have shown that the LD in cells infected with M. leprae are found in close proximity to, attached to and even internalised into the parasitecontaining vacuole (Fig. 1D). Schwann cells also exhibit a relocalisation of adipose differentiation related protein (ADRP)-positive LD to bacteria-containing phagosomes in cultured cells and in nerve biopsies from infected patients (Fig. 1E, F) (Mattos et al. 2011a). This lipid droplet relocalisation depends on PI3K signalling and requires cytoskeletal rearrangements (Mattos et al. 2011a) and blocking lipid droplet movement by interfering with cytoskeleton function decreases bacterial survival in infected cells (Mattos et al. 2011a). These data suggest that $M$. leprae intercepts exocytic vesicles from the secretory pathway and reinforces the lipid droplet organelle as a possible source of host cell-derived lipid nutrients. Electron microscopy suggests that lipid droplet content is found within the bacterial cytoplasm (Fig. 1G). M. bovis BCG and M. tuberculosis-infected cells also display a close association of the mycobacterial phagosome with LD (D'Avila et al. 2006, Peyron et al. 2008) and this close association of LD with the pathogen-containing vacuole is thought to constitute an important mechanism for lipid acquisition in vivo (D'Avila et al. 2006, Peyron et al. 2008, Russell et al. 2009, Daniel et al. 2011). In addition, macrophage LD have been shown to accumulate mycobactin (the lipophilic siderophore of mycobacteria)iron complexes and ferric mycobactin-enriched LDs are found in close contact with phagosomes, suggesting that this organellar association enables the delivery of iron to the mycobacteria (Luo et al. 2005). To reinforce these data, which describe the hijacking of LD by pathogens for their own growth, we utilised experimental TB models of BCG infection to demonstrate that LDs are also frequently found in close proximity to bacteria-containing phagosomes and that the inhibition of lipid droplet formation greatly reduces BCG viability (D'Avila et al. 2006, Almeida et al. 2009), suggesting a potential role for lipid droplet biogenesis and recruitment in lipid acquisition by mycobacteria.

Although we do not have a full appreciation of the carbon sources utilised by mycobacteria during infection, there is genetic evidence that mycobacteria utilise LD as lipid nutritional sources. The possibility that $M$. leprae assimilates fatty acids originating from the neutral lipids and phospholipids found in LD (Cole et al. 2001) is plausible due to the presence of two lipases and one phospholipase encoded by the M. leprae genome. In addition, early metabolic studies (Wheeler 1989, 2003) and recent proteomic analyses (Marques et al. 2008) indicate that fatty acid beta-oxidation is an active pathway in in vivo-grown $M$. leprae, which implies that lipids, rather than carbohydrates, are the dominant carbon substrate mobilised during infection. As such, the most reliable method to date for measuring $M$. leprae viability is based on the assumed ability of $M$. leprae to oxidise fatty acids (Franzblau 1988). The role of lipids in M. leprae metabolism is further supported by an active glyoxylate cycle (Marques et al. 2008), referred to as the dominant anaplerotic pathway during fatty acid growth. Furthermore, deletions of the genes required for growth that utilise lipids as a sole carbon source render $M$. leprae bacteria incapable of growth in vivo. Therefore, the utilisation and manipulation of host lipids and lipid pathways represent a significant mechanism for these bacteria to cause and sustain infection.

In turn, the re-alignment of the host metabolism appears to require alterations in the mycobacterial pathogens $M$. tuberculosis and M. leprae, allowing them to switch to the utilisation of lipids as alternative carbon sources. This is best illustrated by M. tuberculosis, which uses a large portion of its coding capacity for the production of enzymes involved in lipogenesis and lipolysis (Cole 1998). Controversially, many of these functional genes found in M. tuberculosis are lacking in M. leprae (Cole 2001). The loss of these genes has caused M. leprae to rely on the host cell to survive: because of reductive evolution, $M$. leprae can only survive in extremely selective environments. The intracellular mycobacteria likely derive much of their energy from the degradation of host-derived lipids, a process initiated by lipases. Interestingly, M. leprae is deficient in many enzymes. In addition, enzymes from the host cell, in fact, may be complementing the bacterial genes that are present, as was recently suggested by a study describing the overexpression of lipases and phospholipases in LL lesions (Cruz et al. 2008). 
Notably, two reports have implicated lipid droplet formation and the intimate association of M. tuberculosis-containing phagosomes in the capacity of bacteria to persist in a long-term dormant state within the human host and avoid anti-mycobacterial drugs (Peyron et al. 2008, Daniel et al. 2011). These data suggest an interesting additional role for host lipids as efficient, hydrophobic, safe shelters. It is possible that lipid droplet recruitment constitutes a prime example of "organelle mimicry", whereby M. leprae successfully escapes host recognition by cloaking itself in these fat-rich structures. This concept has not been excluded as an alternative cellular function of the observed LD in leprosy.

Host LD facilitate the production of lipid immunomodulators and cytokines - Accumulating evidence has indicated that LDs are key inflammation-related organelles with major roles in eicosanoid production (reviewed in Bozza et al. 2011). As such, the role of this organelle in the regulation of the host response to infection via the modulation of the production of inflammatory mediators has been investigated.

Substantial progress has been made in demonstrating that eicosanoid synthesis can occur at newly formed LD sites during mycobacterial infections (D'Avila et al. 2006, 2008b, Mattos et al. 2010, 2011b). Three main classes of downstream products (eicosanoids) can be produced: prostaglandins (PGs), such as $\mathrm{PGE}_{2}$, leukotrienes (LTs),

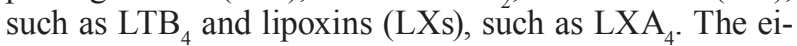
cosanoids synthesised at lipid droplet sites, particularly $\mathrm{PGE}_{2}$, may function as proinflammatory molecules or potent inhibitors of many aspects of the innate and adaptive immune systems (Weissmann 1993, Dooper et al. 2002).

The role of lipid droplet-derived eicosanoids may vary depending on the cell type, stimulus and inflammatory conditions controlling their synthesis (reviewed in Bozza et al. 2011). The foamy macrophages located in areas of pneumonic M. tuberculosis infection in mice have been shown to be positive for cyclooxigenase (COX)-2 staining (Moreno et al. 2002). Definitive evidence for LD as sites of $\mathrm{PGE}_{2}$ synthesis in mycobacterial infection has been provided by demonstrating that COX-2 co-localises with ADRP in BCG-induced LD and that $\mathrm{PGE}_{2}$ forms at LD using the EicosaCell assay (D'Avila et al. 2006). Thus, the enhanced capacity of macrophages to generate $\mathrm{PGE}_{2}$ during infection occurs, at least in part, due to increased lipid droplet biogenesis and the compartmentalisation of eicosanoid production signalling within LD. However, TB offers an intriguing example of the dichotomous characterisation of eicosanoid functions in infection. Infection by M. tuberculosis causes microdisruptions in cell membranes that would normally lead to necrosis, but $\mathrm{PGE}_{2}$, which is abundantly generated within $\mathrm{LD}$, may promote membrane repair to protect against necrosis. Conversely, virulent strains induce the expression of $\mathrm{LXA}_{4}$, which inhibits $\mathrm{PGE}_{2}$, allowing necrosis and, thus, the liberation of pathogens; these bacteria can then invade other cells, disseminating the infection (Chen et al. 2008). $\mathrm{PGE}_{2}$ production that depends on $\mathrm{BCG}$-induced lipid droplet formation may favour bacterial survival in BCG-infected macrophages by promoting the downregulation of the innate immune response, suggesting that the accumulation of LD in infected cells may support bacterial growth and persistence in the host. In addition, BCG has also been shown to induce $\mathrm{LTB}_{4}$ in a manner that is closely related to BCG-induced lipid droplet formation (D'Avila et al. 2006). Supporting a direct correlation between lipid droplet formation and eicosanoid production, M. smegmatis fails to induce this organelle and is unable to generate increased amounts of $\mathrm{PGE}_{2}$, reinforcing the notion of LD as the sites of eicosanoid production and their role in intracellular bacterial survival.

In leprosy, $\mathrm{PGE}_{2}$ production correlates with immunosuppressive effects and a switch to the Th2 immune response on the LL pole. Thus, we have evaluated whether lipid droplet induction correlates with an increase in the cellular production of eicosanoids during $M$. leprae infection. Our laboratory has recently demonstrated that the LD that form in response to M. leprae constitute sites of eicosanoid synthesis, ultimately leading to the increased production of $\mathrm{PGE}_{2}$ by infected macrophages and Schwann cells (Mattos et al. 2010, 2011b). The experimental conditions that inhibit M. leprae-induced lipid droplet formation also downregulate M. leprae-induced $\mathrm{PGE}$, secretion, supporting the notion that LDs are important for $\mathrm{PGE}_{2}$ biosynthesis in M. leprae-infected cells. The colocalisation of ADRP, COX-2 and M. leprae inside Schwann cells in nerve biopsies from LL patients strongly indicates that M. leprae-induced LDs are intracellular sites of eicosanoid synthesis in vivo (Fig. 1H) (Mattos et al. 2011b). Collectively, these data support the role of LD as dynamic organelles involved in the production of inflammatory mediators.

It has been shown that the high $\mathrm{PGE}_{2}$ concentrations produced by COX-2-positive macrophages in LL are able to inhibit Th1 cytokine production and, accordingly, most likely contribute to T-cell anergy at this disease pole (Fig. 1I) (Betz \& Fox 1991, Kiszewski et al. 2003). However, lipid droplet-dependent $\mathrm{PGE}_{2}$ synthesis might account for the persistence of $M$. leprae in infected macrophages and Schwann cells when compared with M. tuberculosis infection. The notion that lipid droplet formation in foamy cells is not only a characteristic feature of LL, but is also a key pathophysiological mechanism that inhibits the development of a robust Th1 response and the eradication of the pathogen is now well supported.

In mycobacterial infections, lipid droplet formation is associated with alterations in the expression of inflammatory cytokines. The evidence that the pharmacological inhibition of lipid droplet formation by C-75, a fatty acid synthase (FAS) inhibitor and NS-398, a selective COX-2 inhibitor, can revert BCG-induced proinflammatory cytokine production (D'Avila et al. 2008b) supports the notion of a link between lipid droplet formation and an altered cytokine profile. In different cell systems, lipid droplet biogenesis has been shown to require the synthesis of new lipids via a mechanism that is tightly regulated by FAS (Schmid et al. 2005, Accioly et al. 2008, Samsa et al. 2009). Although C-75 is not a COX inhibitor, C-75-induced lipid droplet inhibition leads to the inhibition of $\mathrm{PGE}_{2}$ production. Accordingly, C-75 inhibits mycobacteria-infected macrophages and subcapsular sinus (SCs) through mechanisms that are 
dependent on lipid droplet inhibition and independent of COX-2 inhibition (D'Avila et al. 2008b, Mattos et al. 2011b). In addition, treatment with NS-398 was found to exert COX-2-independent inhibitory effects on the lipid droplet biogenesis induced by $M$. leprae infection, which occurs in parallel with the ability of NS-398 to inhibit cyclooxygenase. These data reinforce the role of lipid metabolism in modulating the innate immune response triggered by M. leprae infection in SCs via lipid droplet formation (Mattos et al. 2011b).

The potential role of LD as immunomodulatory platforms for cytokine regulation in foamy M. leprae-infected cells has also been investigated. Studies have analysed inflammatory mediators and it has been shown that infected cells secrete increased levels of the anti-inflammatory cytokine IL-10 and reduced levels of IL-12 and nitric oxide compared to the basal levels observed in uninfected cells. Interestingly, when comparing conditions that induce $(M$. leprae infection) and impair (M. leprae infection pretreated with cytochalasin, C-75, NS398) lipid droplet formation, we observed a decrease in IL-10 production and an increase in IL-12 and nitrite detection in the culture supernatants, suggesting that LD may negatively modulate the immune response of host cells to intracellular infection (Mattos et al. 2010, 2011b). Additionally, we found an inhibition of $\mathrm{PGE}_{2}$ production when drugs were used to impair lipid droplet biogenesis. $\mathrm{PGE}_{2}$ is a potent immune modulator that downregulates Th1 responses and bactericidal activity toward intracellular organisms (Renz et al. 1988, Betz \& Fox 1991, van der Pouw Kraan et al. 1995, D'Avila et al. 2006). Curiously, LDs are also described as sites of cytokine storage, including tumour necrosis factor, macrophage migration inhibitory factor, fibroblast growth factor and regulated on activation, normal $\mathrm{T}$ cell expressed and secreted in activated leukocytes (Beil et al. 1995, Lim et al. 1996, Dvorak et al. 2001, Pacheco et al. 2002, Assunção-Miranda et al. 2010). Thus, the signalling pathways that regulate the immune cascade in M. lepraeinfected cells and the following potential mechanisms deserve further investigation: (i) high IL-10 production involves an autocrine $\mathrm{PGE}_{2}$ circuit, as has been shown in the context of other cell types (Alvarez et al. 2010) and (ii) lipid droplet-stored cytokines may function locally as intracrine signalling mediators (Figs 1,2).

Persistence and viability - The interaction between intracellular bacteria and their host deserves special attention because obligate intracellular bacteria, such as M. tuberculosis and M. leprae, need to acquire resources from their hosts to survive, replicate and persist.

Recently, the impact of lipid droplet biogenesis inhibition by NS-398 or C-75 on the killing capacity of cells infected by M. leprae has been investigated. Strikingly, we have demonstrated that treatment with these pharmacological inhibitors favours mycobacterial killing by Schwann cells in addition to the blockage of lipid droplet biogenesis (Mattos et al. 2011b), suggesting a role for newly formed LD in intracellular M. leprae survival within nerves. In support of the hypothesis that LDs are involved in $M$. leprae survival, pathogen-induced host lipid droplet formation has been associated with advan- tages in intracellular pathogen survival during hepatitis $\mathrm{C}$ virus (Boulant et al. 2007, Miyanari et al. 2007), dengue virus (Samsa et al. 2009), Trypanosoma cruzi (D'Avila et al. 2011) and BCG (D'Avila et al. 2008b, Almeida et al. 2009) infections. In addition to lipid droplet biogenesis, $M$. leprae is able to recruit LD promptly during bacterial attachment and internalisation in the bacteria-containing phagosomes in which they accumulate (Mattos et al. 2011a). Because LDs are organelles that are rich in neutral lipids, we investigated whether lipid droplet recruitment to bacterial phagosomes favours mycobacterial survival. To this end, the cells were infected with $M$. leprae and then treated with cytochalasin D or Taxol to inhibit lipid droplet trafficking. We demonstrated that LDs are promptly recruited to bacteria-containing phagosomes during M. leprae internalisation in Schwann cells and that this event contributes to bacterial survival inside the cells (Mattos et al. 2011a). In addition, it has been suggested that LDs are a rich source of nutrients for mycobacterial intracellular growth (D'Avila et al. 2006, Peyron et al. 2008, Almeida et al. 2009, Cáceres et al. 2009, Russell et al. 2009). Thus, the contribution of lipid droplet inhibition to improvements in the killing capacity of M. leprae-infected Schwann cells may occur as a secondary effect of the inhibition of $\mathrm{PGE}_{2}$ production and modulation of the cytokine milieu.

Future directions - In conclusion, the in vivo and in vitro data presented here show that $M$. leprae triggers a pronounced alteration in host-cell lipid homeostasis through the regulation of lipid droplet biogenesis and the recruitment of these organelles to bacterial phagosomes. This modulation of LD contributes to the foamy phenotype of infected cells and favours intracellular bacterial survival, likely representing a fundamental aspect of $M$. leprae pathogenesis. The comparative analyses of the changes in the host cell lipidome with different mycobacterial species illustrate the unique characteristics of each particular pathogen in a cell type-dependent mechanism (Table).

A model can be proposed in which the lipid storage phenomenon observed in M. leprae-infected cells plays a key role in leprosy pathogenesis by facilitating bacterial persistence in the host via two or more mechanisms (Fig. 2). The significance of the redistribution of host LD that is triggered by $M$. leprae remains to be established, but some intriguing data are emerging. Lipid droplet recruitment likely constitutes an effective $M$. leprae intracellular strategy for acquiring host cell lipids as a nutritional source and promoting bacterial survival. In turn, the realignment of the host metabolism appears to require alterations in the mycobacterial pathogens themselves (i.e., M. tuberculosis and M. leprae): the bacteria switch to the utilisation of lipids as alternative carbon sources. Although we do not have a full appreciation of the carbon sources utilised by the infecting mycobacteria, there is genetic evidence that mycobacteria utilise the $\mathrm{LD}$ as a nutritional source. However, we cannot exclude the additional possibility that lipid droplet sequestration by bacteria-containing phagosomes provides a temporary shelter for M. leprae. 
Additionally, the accumulation of host-derived lipids in infected Schwann cells favours the generation of an innate immune response that may contribute to a permissive environment for $M$. leprae proliferation within LL nerves/skin. M. leprae-infected cells also produce IL-10, which, together with $\mathrm{PGE}_{2}$, favours the inhibition of cell bactericidal activities and the downregulation of Th1-type cytokines. Thus, the suppression of normal inflammatory functions by M. leprae in macrophages and Schwann cells results in the expression of an anti-inflammatory phenotype that is dependent on lipid droplet biogenesis.

Pathogens use lipids as building blocks and a nutrient source and also to influence the host cell physiology to enable their own survival and replication. The data indicate that multibacillary M. leprae infection is associated with a dramatic induction of host lipid metabolism genes, including multiple lipases with functional domains that were predicted by a comparative domain analysis to complement the deficiencies of the pathogen. In particular, the study of lipid droplet functions during host-pathogen interactions will provide a great opportunity for future research and can be expected to afford opportunities to profile these host-pathogen interactions and to study their important contributions to the ability of pathogens to escape from the immune system.

Lipid profiling may become an important method to determine the metabolic condition of a cell or organism. The specific depletion, presence or modulation of certain lipid species may generate lipid fingerprints that are unique to infection by specific pathogens, thus establishing biomarkers for these pathogens. Clearly, such an underlying regulatory mechanism may hold the key to the development of novel strategies aimed at preventing or, at a minimum, limiting peripheral nerve injury in leprosy. The combination of epidemiological and biological evidence suggests that leprosy cannot be eliminated by MDT alone. Indeed, M. leprae makes extensive use of the host cell lipidome and has evolved very sophisticated mechanisms to utilise the diversity and complexity of host LD to maximise its own survival advantage. As such, strategies to inhibit lipid droplet formation in leprosy may represent novel opportunities for the development of adjunctive anti-leprosy therapies. Thus, we believe that the findings highlighted here only scratch the surface of this rich new area of investigation into lipid droplet biology and its potential use in therapies.

\section{REFERENCES}

Accioly MT, Pacheco P, Maya-Monteiro CM, Carrossini N, Robbs BK, Oliveira SS, Kaufmann C, Morgado-Diaz JA, Bozza PT, Viola JP 2008. Lipid bodies are reservoirs of cyclooxygenase-2 and sites of prostaglandin- $\mathrm{E}_{2}$ synthesis in colon cancer cells. Cancer Res 68: 1732-1740.

Alcais A, Mira M, Casanova J-L, Schurr E, Abel L 2005. Genetic dissection of immunity in leprosy. Curr Opin Immunol 17: 44-48.

Almeida PE, Silva AR, Maya-Monteiro CM, Töröcsik D, D’Avila H, Dezsö B, Magalhães KG, Castro-Faria-Neto HC, Nagy L, Bozza PT 2009. Mycobacterium bovis bacillus Calmette-Guérin infection induces TLR2-dependent peroxisome proliferator-activated receptor-expression and activation: functions in inflammation, lipid metabolism and pathogenesis. J Immunol 183: 1337-1345.
Alvarez S, Blanco A, Fresno M, Munõz-Fernández MA 2010. Nuclear factor-kappaB activation regulates cyclooxygenase-2 induction in human astrocytes in response to CXCL12: role in neuronal toxicity. J Neurochem 113: 772-783.

Assunção-Miranda I, Amaral FA, Bozza FA, Fagundes CT, Sousa LP, Souza DG, Pacheco P, Barbosa-Lima G, Gomes RN, Bozza PT, Da Poian AT, Teixeira MM, Bozza MT 2010. Contribution of macrophage migration inhibitory factor to the pathogenesis of dengue virus infection. FASEB J 24: 218-228.

Beil WJ, Weller PF, Peppercorn MA, Galli SJ, Dvorak AM 1995. U1trastructural immunogold localization of subcellular sites of TNFalpha in colonic Crohn's disease. J Leukoc Biol 58: 284-298.

Betz M, Fox BS 1991. Prostaglandin $\mathrm{E}_{2}$ inhibits production of Th1 lymphokines, but not of Th2 lymphokines. J Immunol 146: 108-113.

Boulant S, Targett-Adams P, McLauchlan J 2007. Disrupting the association of hepatitis $\mathrm{C}$ virus core protein with lipid droplets correlates with a loss in production of infectious virus. J Gen Virol 88: 2204-2213.

Bozza PT, Bakker-Abreu I, Navarro-Xavier RA, Bandeira-Melo C 2011. Lipid body function in eicosanoid synthesis: an update. Prostaglandins Leukot Essent Fatty Acids 85: 205-213.

Bozza PT, D’Avila H, Almeida PE, Magalhães KG, Molinaro R, Almeida CJ, Maya-Monteiro CM 2009a. Lipid droplets in hostpathogen interactions. Clinical lipidology 4: 791-807.

Bozza PT, Magalhães KG, Weller PF 2009b. Leukocyte lipid bodies - Biogenesis and functions in inflammation. Biochim Biophys Acta 1791: 540-551.

Bozza PT, Yu W, Cassara J, Weller PF 1998. Pathways for eosinophil lipid body induction: differing signal transduction in cells from normal and hypereosinophilic subjects. J Leukoc Biol 64: 563-569.

Cáceres N, Tapia G, Ojanguren I, Altare F, Gil O, Pinto S, Vilaplana C, Cardona PJ 2009. Evolution of foamy macrophages in the pulmonary granulomas of experimental tuberculosis models. Tuberculosis 89: 175-182.

Cao F, Castrillo A, Tontonoz P, Re F, Byrne GI 2007. Chlamydia pneumoniae-induced macrophage foam cell formation is mediated by Toll-like receptor 2. Infect Immun 75: 753-759.

Cardoso CC, Pereira AC, de Sales Marques C, Moraes MO 2011. Leprosy susceptibility: genetic variations regulate innate and adaptive immunity and disease outcome. Future Microbiol 6: 533-549.

Chatterjee KR, Das Gupta NN, De ML 1959. Electron microscopic observations on the morphology of Mycobacterium leprae. Exp Cell Res 18: 521-527.

Chen M, Divangahi M, Gan H, Shin DS, Hong S, Lee DM, Serhan CN, Behar SM, Remold HG 2008. Lipid mediators in innate immunity against tuberculosis: opposing roles of $\mathrm{PGE}_{2}$ and $\mathrm{LXA}_{4}$ in the induction of macrophage death. J Exp Med Nov 205: 2791-2801.

Cole ST, Eiglmeier K, Parkhill J, James KD, Thomson NR, Wheeler PR, Honoré N, Garnier T, Churcher C, Harris D, Mungall $\mathrm{K}$, Basham D, Brown D, Chillingworth T, Connor R, Davies RM, Devlin K, Duthoy S, Feltwell T, Fraser A, Hamlin N, Holroyd S, Hornsby T, Jagels K, Lacroix C, Maclean J, Moule S, Murphy L, Oliver K, Quail MA, Rajandream MA, Rutherford KM, Rutter S, Seeger K, Simon S, Simmonds M, Skelton J, Squares R, Squares S, Stevens K, Taylor K, Whitehead S, Woodward JR, Barrell BG 2001. Massive gene decay in the leprosy bacillus. Nature 409: 1007-1011.

Cole ST, Brosch R, Parkhill J, Garnier T, Churcher C, Harris D, Gordon SV, Eiglmeier K, Gas S, Barry CE 3rd, Tekaia F, Badcock K, Basham D, Brown D, Chillingworth T, Connor R, Davies R, Devlin K, Feltwell T, Gentles S, Hamlin N, Holroyd S, Hornsby 
T, Jagels K, Krogh A, McLean J, Moule S, Murphy L, Oliver K, Osborne J, Quail MA, Rajandream MA, Rogers J, Rutter S, Seeger K, Skelton J, Squares R, Squares S, Sulston JE, Taylor K, Whitehead S, Barrell BG 1998. Deciphering the biology of Mycobacterium tuberculosis from the complete genome sequence. Nature 9: 1089-1097.

Cruz D, Watson AD, Miller CS, Montoya D, Ochoa MT, Sieling PA, Gutierrez MA, Navab M, Reddy ST, Witztum JL, Fogelman AM, Rea TH, Eisenberg D, Berliner J, Modlin RL 2008. Host-derived oxidized phospholipids and HDL regulate innate immunity in human leprosy. J Clin Invest 118: 2917-2928.

D’Avila H, Freire-de-Lima CG, Roque NR, Teixeira L, Barja-Fidalgo C, Silva AR, Melo RC, dos Reis GA, Castro Faria Neto HC, Bozza PT 2011. Host cell lipid bodies triggered by Trypanosoma cruzi infection and enhanced by the uptake of apoptotic cells are associated with $\mathrm{PGE}_{2}$ generation and increased parasite growth. J Infect Dis 204: 951-961.

D’Avila H, Maya-Monteiro CM, Bozza PT 2008a. Lipid bodies in innate immune response to bacterial and parasite infections. Int Immunopharmacol 8: 1308-1315.

D'Avila H, Melo RCN, Parreira GG, Werneck-Barroso E, CastroFaria-Neto HC, Bozza PT 2006. Mycobacterium bovis bacillus Calmette-Guérin induces TLR2-mediated formation of LBs: intracellular domains for eicosanoid synthesis in vivo. J Immunol 176: 3087-3097.

D'Avila H, Roque NR, Cardoso RM, Castro-Faria-Neto HC, Melo RC, Bozza PT 2008b. Neutrophils recruited to the site of Mycobacterium bovis BCG infection undergo apoptosis and modulate lipid body biogenesis and prostaglandin E production by macrophages. Cell Microbiol 10: 2589-2604.

Daniel J, Maamar H, Deb C, Sirakova TD, Kolattukudy PE 2011. Mycobacterium tuberculosis uses host triacylglycerol to accumulate lipid droplets and acquires a dormancy-like phenotype in lipidloaded macrophages. PLoS Pathog 7 (Suppl.): S1-S16.

de Messias-Reason I, Kremsner PG, Kun JF 2009. Functional haplotypes that produce normal ficolin-2 levels protect against clinical leprosy. J Infect Dis 199: 801-804.

Dooper MM, Wassink L, M'Rabet L, Graus YM 2002. The modulatory effects of prostaglandin-E on cytokine production by human peripheral blood mononuclear cells are independent of the prostaglandin subtype. Immunology 107: 152-159.

Dvorak AM, Morgan ES, Weller PF 2001. Ultrastructural immunolocalization of basic fibroblast growth factor to lipid bodies and secretory granules in human mast cells. Histochem J 33: 397-402.

Franzblau SG 1988. Oxidation of palmitic acid by Mycobacterium leprae in an axenic medium. J Clin Microbiol 26: 18-21.

Halvorsen B, Wæhre T, Scholz H, Clausen OP, von der Thüsen JH, Müller F, Heimli H, Tonstad S, Hall C, Frøland SS, Biessen EA, Damås JK, Aukrust P 2005. Interleukin-10 enhances the oxidized LDL-induced foam cell formation of macrophages by antiapoptotic mechanisms. J Lipid Res 46: 211-219.

Job CK 1970. Mycobacterium leprae in nerve lesions in lepromatous leprosy: an electron microscopic study. Arch Pathol 89: 195-207.

Kazemi MR, McDonald CM, Shigenaga JK, Grunfeld C, Feingold KR 2005. Adipocyte fatty acid-binding protein expression and lipid accumulation are increased during activation of murine macrophages by Toll-like receptor agonists. Arterioscler Thromb Vasc Biol 25: 1220-1224.

Kim MJ, Wainwright HC, Locketz M, Bekker LG, Walther GB, Dittrich C, Visser A, Wang W, Hsu FF, Wiehart U, Tsenova L, Kaplan G, Russell DG 2010. Caseation of human tuberculo- sis granulomas correlates with elevated host lipid metabolism. EMBO Mol Med 2: 258-274.

Kiszewski AE, Becerril E, Baquera J, Ruiz-Maldonado R, Hernández Pando R 2003. Expression of cyclooxygenase type 2 in lepromatous and tuberculoid leprosy lesions. Br J Dermatol 148: 795-798.

Krutzik SR, Ochoa MT, Sieling PA, Uematsu S, Ng YW, Legaspi A, Liu PT, Cole ST, Godowski PJ, Maeda Y, Sarno EN, Norgard MV, Brennan PJ, Akira S, Rea TH, Modlin RL 2003. Activation and regulation of Toll-like receptors 2 and 1 in human leprosy. Nat Med 9: 525-532.

Kurup G, Mahadevan PR 1982. Cholesterol metabolism of macrophages in relation to the presence of Mycobacterium leprae. J Biosci 4: 307-316.

Lim KG, Wan HC, Bozza PT, Resnick MB, Wong DT, Cruikshank WW, Kornfeld H, Center DM, Weller PF 1996. Human eosinophils elaborate the lymphocyte chemoattractants. IL-16 (lymphocyte chemoattractant factor) and RANTES. J Immunol 156: 2566-2570.

Luo M, Fadeev EA, Groves JT 2005. Mycobactin-mediated iron acquisition within macrophages. Nat Chem Biol 1: 149-153.

Marques MA, Neves-Ferreira AG, da Silveira EK, Valente RH, Chapeaurouge A, Perales J, da Silva BR, Dobos KM, Spencer JS, Brennan PJ, Pessolani MC 2008. Deciphering the proteomic profile of Mycobacterium leprae cell envelope. Proteomics 8: 2477-2491.

Mattos KA, D'Avila H, Rodrigues LS, Oliveira VG, Sarno EN, Atella GC, Pereira GM, Bozza PT, Pessolani MC 2010. Lipid droplet formation in leprosy: Toll-like receptor-regulated organelles involved in eicosanoid formation and Mycobacterium leprae pathogenesis. J Leukoc Biol 87: 371-384.

Mattos KA, Lara FA, Oliveira VG, Rodrigues LS, D’Avila H, Melo RC, Manso PP, Sarno EN, Bozza PT, Pessolani MC 2011a. Modulation of lipid droplets by Mycobacterium leprae in Schwann cells: a putative mechanism for host lipid acquisition and bacterial survival in phagosomes. Cell Microbiol 13: 259-273.

Mattos KA, Oliveira VG, D'Avila H, Rodrigues LS, Pinheiro RO, Sarno EN, Pessolani MC, Bozza PT 2011b. TLR6-driven lipid droplets in Mycobacterium leprae-infected Schwann cells: immunoinflammatory platforms associated with bacterial persistence. J Immunol 187: 2548-2558.

Means TK, Wang S, Lien E, Yoshimura A, Golenbock DT, Fenton MJ 1999. Human Toll-like receptors mediate cellular activation by Mycobacterium tuberculosis. J Immunol 163: 3920-3927.

Miyanari Y, Atsuzawa K, Usuda N, Watashi K, Hishiki T, Zayas M, Bartenschlager R, Wakita T, Hijikata M, Shimotohno K 2007. The lipid droplet is an important organelle for hepatitis $\mathrm{C}$ virus production. Nat Cell Biol 9: 1089-1097.

Montoya D, Cruz D, Teles RM, Lee DJ, Ochoa MT, Krutzik SR, Chun R, Schenk M, Zhang X, Ferguson BG, Burdick AE, Sarno EN, Rea TH, Hewison M, Adams JS, Cheng G, Modlin RL 2009. Divergence of macrophage phagocytic and antimicrobial programs in leprosy. Cell Host Microbe 6: 343-353.

Moreno C, Rees AJ 1993. Striking the right balance; the role of cytokines in mycobacterial disease. Clin Exp Immunol 94: 1-3.

Moreno JR, Garcia IE, Hernandez MLG, Leon DA, Marquez R, Pando RH 2002. The role of prostaglandin $E_{2}$ in the immunopathogenesis of experimental pulmonary tuberculosis. Immunology 106: 257-266.

Oliveira RB, Ochoa MT, Sieling PA, Rea TH, Rambukkana A, Sarno EN, Modlin RL 2003. Expression of Toll-like receptor 2 on human Schwann cells: a mechanism of nerve damage in leprosy. Infect Immun 71: 1427-1433. 
Pacheco P, Bozza FA, Gomes RN, Bozza M, Weller PF, Castro-Faria-Neto HC, Bozza PT 2002. Lipopolysaccharide-induced leukocyte lipid body formation in vivo: innate immunity elicited intracellular loci involved in eicosanoid metabolism. J Immunol 169: 6498-6506.

Pacheco P, Vieira-de-Abreu A, Gomes RN, Barbosa-Lima G, Wermelinger LB, Maya-Monteiro CM, Silva AR, Bozza MT, Castro-Faria-Neto HC, Bandeira-Melo C, Bozza PT 2007. Monocyte chemoattractant protein-1/CC chemokine ligand 2 controls microtubule-driven biogenesis and leukotriene $\mathrm{B}_{4}$-synthesizing function of macrophage lipid bodies elicited by innate immune response. J Immunol 179: 8500-8508.

Parkash O 2009. Classification of leprosy into multibacillary and paucibacillary groups: an analysis. FEMS Immunol Med Microbiol 55: 1-5.

Persson J, Nilsson J, Lindholm MW 2008. Interleukin-1 $\beta$ and tumor necrosis factor- $\alpha$ impede neutral lipid turnover in macrophagederived foam cells. BMC Immunol 25: 70 .

Peyron P, Vaubourgeix J, Poquet Y, Levillain F, Botanch C, Bardou F, Daffé M, Emile JF, Marchou B, Cardona PJ, de Chastellier C, Altare F 2008. Foamy macrophages from tuberculous patients' granulomas constitute a nutrient-rich reservoir for M. tuberculosis persistence. PLoS Pathog 4: e1000204.

Renz, H, Gong JH, Schmidt A, Nain M, Gemsa D 1988. Release of tumor necrosis factor-alpha from macrophages. Enhancement and suppression are dose-dependently regulated by prostaglandin $\mathrm{E}_{2}$ and cyclic nucleotides. J Immunol 141: 2388-2393.

Ridley DS 1974. Histological classification and the immunological spectrum of leprosy. Bull World Health Organ 51: 451-465.

Russell DG, Cardona PJ, Kim MJ, Allain S, Altare F 2009. Foamy macrophages and the progression of the human tuberculosis granuloma. Nat Immunol 10: 943-948.

Saka HA, Valdivia RH 2012. Emerging roles for lipid droplets in immunity and host-pathogen interactions. Annu Rev Cell Dev Biol: 411-437.

Sakurai I, Skinsnes OK 1970. Lipids in leprosy 2. Histochemistry of lipids in human leprosy. Int J Lepr Other Mycobact Dis 38: 389-403.

Samsa MM, Mondotte JA, Iglesias NG, Assunção-Miranda I, Barbosa-Lima G, da Poian AT, Bozza PT, Gamarnik AV 2009. Dengue virus capsid protein usurps lipid droplets for viral particle formation. PLoS Pathog 5: e1000632.
Schmid B, Rippmann JF, Tadayyon M, Hamilton BS 2005. Inhibition of fatty acid synthase prevents preadipocyte differentiation. Biochem Biophys Res Commun 328: 1073-1082.

Stewart CR, Stuart LM, Wilkinson K, van Gils J M, Deng J, Halle A, Rayner KJ, Boyer L, Zhong R, Frazier WA, Frazier WA, LacyHulbert A, El Khoury J, Golenbock DT, Moore KJ 2010. CD36 ligands promote sterile inflammation through assembly of a Tolllike receptor 4 and 6 heterodimer. Nat Immunol 11: 155-161.

Takeuchi O, Sato S, Horiuchi T, Hoshino K, Takeda K, Dong Z, Modlin RL, Akira S 2002. Cutting edge: role of Toll-like receptor 1 in mediating immune response to microbial lipoproteins. J Immunol 169: 10-14.

Tanigawa K, Suzuki K, Nakamura K, Akama T, Kawashima A, Wu H, Hayashi M, Takahashi S, Ikuyama S, Ito T, Ishii N 2008. Expression of adipose differentiation-related protein (ADRP) and perilipin in macrophages infected with Mycobacterium leprae. FEMS Microbiol Lett 289: 72-79.

van der Meer-Janssen YP, van Galen J, Batenburg JJ, Helms JB 2010. Lipids in host-pathogen interactions: pathogens exploit the complexity of the host cell lipidome. Prog Lipid Res 49: 1-26.

van der Pouw Kraan TC, Boeije LC, Smeenk RJ, Wijdenes J, Aarden LA 1995. Prostaglandin- $\mathrm{E}_{2}$ is a potent inhibitor of human interleukin 12 production. $J$ Exp Med 181: 775-779.

Virchow R 1863. Die krankhaften Geschwu lste, August Hirschwald, Berlin, 208 pp.

Weissmann G 1993. Prostaglandins as modulators rather than mediators of inflammation. J Lipid Mediat 6: 275-286.

Wheeler PR 1989. Biosynthetic pathways in Mycobacterium leprae. Acta Leprol 7: 21-24.

Wheeler PR 2003. Leprosy - clues about the biochemistry of Mycobacterium leprae and its host-dependency from the genome. World J Microbiol Biotechnol 19: 1-16.

WHO - World Health Organization. 2010. Leprosy today. Available from: who. int/lep/en/.

Yamamura M, Uyemura K, Deans RJ, Weinberg K, Rea TH, Bloom BR, Modlin RL 1991. Defining protective responses to pathogens: cytokine profiles in leprosy lesions. Science 254: 277-279.

Zehmer JK, Huang Y, Peng G, Pu J, Anderson RGW, Liu P 2009. A role for lipid droplets in inter-membrane lipid traffic. Proteomics 9: 914-921. 